\title{
Role of PET/CT in Evaluating for Occult Gram-Positive Bacteremia
}

\author{
Jin Ge, MD, MBA and Tuyen Kiet, MD \\ Department of Medicine, University of California, San Francisco, San Francisco, CA, USA.
}

KEY WORDS: infectious disease; clinical images; clinical reasoning. $\mathrm{J}$ Gen Intern Med 32(4):485

DOI: $10.1007 / \mathrm{s} 11606-016-3910-0$

(c) Society of General Internal Medicine 2016

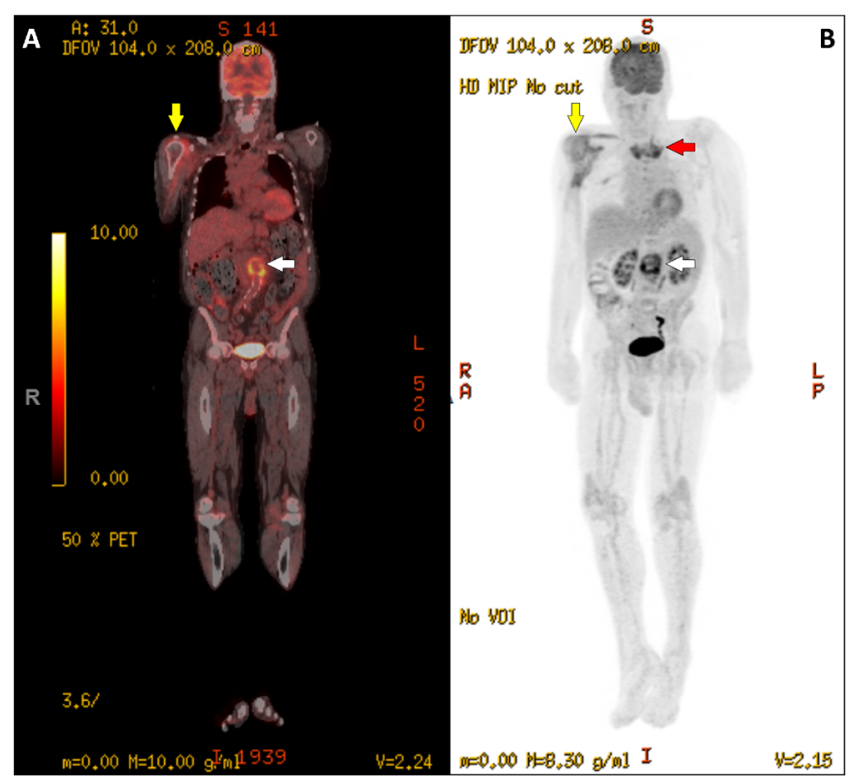

A

67-year-old man with a history of ascending thoracic aneurysm, asthma, and schizophrenia presented with tachypnea after cardiac catheterization. Exam showed increased work of breathing. Blood cultures grew methicillinsusceptible Staphylococcus aureus (MSSA), which was treated with intravenous cefazolin. Despite treatment, the patient remained persistently bacteremic. An exhaustive search for a source proved unfruitful; notably, a transthoracic echocardiogram (TTE) showed no evidence of endocarditis. A whole- body PET/CT was then conducted to evaluate for occult sources. This study showed a T2 vertebrae with osteomyelitis (red arrow), right glenohumeral edema with soft tissue infection (yellow arrows), and a 4.8-cm infrarenal mycotic aortic aneurysm (white arrows).

Although PET/CT is not commonly part of the diagnostic algorithm for bacteremia, it has been shown to be valuable in certain cases. In a 2010 study of PET/CT in occult grampositive bacteremia, this modality was the first to delineate the infectious foci in $30 \%$ of the study patients. ${ }^{1}$ The same study group, based at Radboud University, also performed a cost-effectiveness analysis that showed that routine use of PET/CT in gram-positive bacteremia had an acceptable cost per death prevented in the Netherlands. ${ }^{2}$ This case illustrates the utility of PET/CT as a second-line diagnostic tool in the management of patients with occult bacteremia and fever. ${ }^{3}$

Corresponding Author: Jin Ge, MD, MBA; Department of MedicineUniversity of California, San Francisco, San Francisco, CA, USA (e-mail: Jin.Ge@ucsf.edu).

\section{Compliance with Ethical Standards:}

Conflict of Interest: The authors declare that there are no conflicts of interest.

\section{REFERENCES}

1. Vos FJ, Bleeker-Rovers CP, Sturm PD, et al. 18F-FDG PET/CT for detection of metastatic infection in gram-positive bacteremia. J Nucl Med. 2010;51(8): 1234-1240.

2. Vos FJ, Bleeker-Rovers CP, Kullberg BJ, Adang EM, Oyen WJ. Costeffectiveness of routine (18)F-FDG PET/CT in high-risk patients with gram-positive bacteremia. J Nucl Med. 2011;52(11):1673-1678.

3. Meller J, Sahlmann Co, Scheel AK. 18F-FDG PET and PET/CT in fever of unknown origin. J Nucl Med. 2007;48(1):35-45. 